\title{
PERCEPCIONES ESTUDIANTILES SOBRE LA PRÁCTICA PROFESIONAL DOCENTE EN EDUCACIÓN ARTÍSTICA
}

\section{STUDENT PERCEPTIONS ON TEACHING PROFESSIONAL PRACTICE IN ARTISTIC EDUCATION}

\author{
Napoleón Santiago CARBAJAL LAVADO ${ }^{1}$

\begin{tabular}{|lll|}
\hline Recibido & $:$ & 31.03 .2021 \\
Aceptado & $:$ & 14.07 .2021 \\
Publicado & $:$ & 02.08 .2021 \\
\hline
\end{tabular}

RESUMEN: El propósito de la presente investigación fue develar las concepciones e identificar las rutinas y herramientas metodológicas en la práctica profesional pedagógica en formación docente inicial en la carrera de educación artística, especialidad de artes plásticas. La metodología corresponde a un enfoque mixto en la variedad descriptiva, la recolección de datos se hizo a través de dos técnicas: la escala valorativa de Likert y la asamblea reflexiva, ambos aplicados a los estudiantes de la Escuela Superior de Formación Artística Pública Bellas Artes Macedonio de la Torre de Trujillo, Perú; el análisis se realizó mediante asambleas de sistematización y triangulación. Los resultados obtenidos determinan que las concepciones, prácticas y herramientas corresponden a una práctica profesional pedagógica de tipo técnica, académica o tradicional. La investigación aporta datos a la caracterización de la práctica, y abre la posibilidad de la realización de un nuevo estudio para mejorar dicha situación.

Palabras clave: Concepciones, rutinas, herramientas, práctica profesional, técnica y reflexiva.

\begin{abstract}
The purpose of this research was to reveal the conceptions and identify routines and methodological tools in professional pedagogical practice in inicial teacher training in the specialty of Plastic Arts in the career of artistic education. The methodology corresponds to a mixed approach in the descriptive variety. The data collection was done through two techniques: the Likert rating scale and the reflective assembly, both applied to the students of Escuela Superior de Formación Artística Pública de Bellas Artes Macedonio de La Torre, Trujillo, Peru. The analysis was performed through assemblies of systematization and triangulation. The results determined that the conceptions, practices and tools correspond to a professional pedagogical practice of a technical, academic or traditional type. This research provides data to the characterization of the practice, and opens the possibility of carrying out a new study to improve this situation.
\end{abstract}

Keywords: Conceptions, Routines, Tools, Professional Practice, Technical and Reflective.

\footnotetext{
${ }^{1}$ Doctor en Ciencias de la Educación. Universidad Nacional de Trujillo. Facultad de Educación y Ciencias de la Comunicación. Escuela de Post Grado. Trujillo. Perú. Email: ncarbajal1960@ gmail.com. ORCID: https://orcid.org/00000001-6975-0887
} 


\section{INTRODUCCIÓN}

La presente investigación se desarrolló en la Escuela Superior de Formación Artística Pública Bellas Artes Macedonio de la Torre; los participantes fueron estudiantes de la Carrera Profesional de Educación Artística quienes desarrollaron las asignaturas de práctica docente final IX y X año 2019.

La discusión sobre la formación profesional docente, según Rojas (2015), en los últimos años se ha convertido en una de las principales problemáticas para generar trasformaciones no solo en los docentes, sino también en el propio sistema educativo; en una realidad en la que los procesos de gestión pedagógica y administrativa están inmersas en las profundas metamorfosis sufridas por la sociedad actual y demandan un replanteamiento de las funciones que se requieren del profesor para alcanzar los fines de la educación en este ambiente de cambio.

En este contexto, la formación de docentes de educación artística debe estar orientada a promover la observación, análisis, sistematización, reflexión y crítica de lo que acontece tanto en las aulas, como en las instituciones y los contextos educativos donde desarrollan su práctica profesional.

El modelo del profesor reflexivo, según Domingo (2017), es una opción paradigmática ligada a la educación y el cambio educativo, no solo como un técnico experto, que aplica conocimientos científicos y pedagógicos, sino también como un profesional abierto y flexible a las problemáticas y decisiones que se movilizan en los contextos escolares, y creador de un conocimiento práctico - pedagógico que le permite desarrollar una acción profesional, autónoma, creativa, eficaz y pertinente a la variedad de situaciones educativas en las que tiene que desenvolverse profesionalmente.

La práctica pedagógica reflexiva es la alternativa más adecuada para lograr este propósito, ya que esta posibilita la formación de profesores autónomos, críticos, reflexivos y conscientes de su responsabilidad en la formación integral de los estudiantes que atienden en las instituciones educativas de educación básica regular del país. Es una poderosa herramienta, para fomentar la reflexión crítica de y sobre las prácticas pedagógicas, de las concepciones sobre ellas y de la propia educación, así como de las características de la realidad social que configura el entorno educativo. 


\section{Journal of the Academy | 124 |}

Desde la experiencia como docente formador, al confrontar el marco de referencia expuesto con las actividades que los estudiantes realizan en sus prácticas profesionales intermedias y finales, se ha constatado que manifiestan emociones de temor, inseguridad, dudas, ansiedad e incertidumbre; así como un escaso ejercicio de observación, diagnóstico, sistematización basada en la reflexión de experiencias; grandes dificultades para diseñar propuestas de cambio, tomar decisiones y resolver problemas; situación que desencadena en no implantar cambios profundos y significativos en las aulas e instituciones educativas cuando asumen el rol de docentes.

En el curso de práctica docente final o internado, que es el momento, supuestamente, en el cual los estudiantes deben demostrar sus competencias pedagógicas reflexivas e impulsen innovaciones y cambios en las aulas, por el contrario, se constata escasa innovación en el desarrollo de las mismas; muchas veces terminan asumiendo roles rutinarios e imitando los modelos de enseñanza de los docentes de aula.

Con lo anterior, el presente estudio busca identificar las concepciones, rutinas y herramientas metodológicas que los estudiantes desarrollan en la práctica profesional pedagógica, y determinar si dicha práctica se adscribe en el enfoque técnico o reflexivo.

\section{DESARROLLO}

\section{Ideas claves sobre la práctica profesional reflexiva}

Para Domingo (2015), los rasgos estructurantes de la práctica reflexiva son: "el saber de contenidos", que implica el aprendizaje de los contenidos científicos del área de especialidad, el saber se adquiere durante el estudio de las diferentes disciplinas del currículo. "El saber en la acción y su utilización" que consiste en la aplicación práctica de un conocimiento que, articulado a la experiencia y su dinámica, se vuelve de uso automático en la práctica del quehacer profesional. La "reflexión en la acción" que se manifiesta cuando el estudiante se cuestiona acerca de la eficacia de las estrategias empleadas en la solución de una situación concreta de aprendizaje y replantea su propio saber alcanzado en la acción para experimentar caminos alternativos. Este proceso de aprendizaje es el más complejo debido a que implica reflexionar mientras se actúa; el propósito de la reflexión en este contexto es comprobar la acción misma que a su vez da paso a la reestructuración de las estrategias de acción, de las 


\section{Journal of the Academy $|125|$}

formas de afrontar los problemas y de las ideas que se tienen sobre los hechos y fenómenos: (Salinas et al., 2017).

Primer eje de la práctica reflexiva. La reflexión, entendida no solo como un proceso deliberado de pensamiento, crítico y fundamentado en el examen, argumentación, discusión y toma de decisiones referente a las actuaciones como docentes; sino que también implica componentes éticos que condicionan al docente a desarrollar acciones orientadas hacia los fines educativos de formar integralmente ciudadanos libres, creativos y talentosos para edificar un mundo más solidario y humano (Cárdenas, 2015).

La reflexión, debe ser entendida como un proceso recursivo y en espiral, de idas y regresos, como un conjunto de destellos de pensar, sentir y actuar. El proceso reflexivo se afirma en lo vivido, en la experiencia vital del docente, quien indaga, profundiza y busca en ella comprensiones que, en lugar de reflejar de forma mecánica una imagen fotográfica de los detalles, cambian la dirección y mirada para abrir nuevas formas y caminos. Por lo tanto, reflexionar implica seguir un camino que parte de la experiencia, donde los saberes y el conocimiento se ponen al servicio de esta como elementos secundarios que facilitan el pensar y no como verdades teóricas a aplicar. La reflexión siempre sigue un camino o vía inductiva, en la que el saber y el conocimiento son utilizados para clarificar, interpretar, profundizar y cambiar el punto de vista, mas no para crear dependencia de ellos (Souto, 2016).

Segundo eje. La crítica, es complementaria a la reflexión y consiste en emitir juicios, razonamientos, opiniones y argumentaciones basados en criterios; en razonar y evaluar lo discutido acerca de temas, situaciones o problemas. Es la capacidad que tiene el docente de autocorregirse, reconociendo sus debilidades (Carbajal, 2013).

Tercer eje. Las concepciones son procesos personales a través de los cuales un individuo estructura su saber a medida que integra y articula sus conocimientos. Este saber se construye, durante un período bastante amplio de la vida a partir de la práctica social y cultural parental del niño en la escuela, de la influencia de los medios de comunicación social en un segundo momento y, finalmente, por el ejercicio profesional y social del adulto. Por consiguiente, las concepciones tienen raíces sociales y culturales, que una vez consolidadas, es un factor de socialización que se concretiza en los intercambios sociales producidos en el campo de la acción y la práctica (Lupion y Gallego, 2017). 
En el contexto de las concepciones, Ávila y Pasek (2013) sostiene que el profesor posee una trayectoria cultural resultado de sus múltiples interacciones, no solo con los seres humanos sino con diversos dispositivos tecnológicos y socioculturales a través de los cuales construye sus percepciones, visiones y creencias de la vida, de la educación, del mundo y de sí mismo; creencias que a lo largo del tiempo se van arraigando en su conciencia y se constituyen en su identidad personal y profesional, es decir en sus concepciones.

El profesor reflexivo es el profesional que asume que la generación del nuevo conocimiento acerca de la enseñanza y aprendizaje es una de sus responsabilidades y posibilidades, poniendo en juego sus concepciones, ideas, creencias, experiencias y teorías.

Anijovich y Capelletti (2018), postulan que para formar docentes reflexivos se debe articular los siguientes procesos: registro de incidentes críticos, plantear preguntas e incertidumbres, realizar rutinas de revisión de prácticas y observación entre pares. La formación de docentes reflexivos se debe sustentar en la reflexión y análisis de la práctica profesional como estrategia de trabajo colectivo, orientado a desarrollar la competencia de aprender a aprender, aprender a desaprender en diálogo, autoanálisis y autorregulación, a través de conocimientos, habilidades y actitudes que le permita convertirse en un docente inquieto e innovador que aprenda de sus aciertos y errores (Lever y López, 2019).

Finalmente, el profesor como un profesional reflexivo, es capaz de generar conocimiento pedagógico práctico a través de la reflexión, a partir de su propia práctica de la enseñanza; pero esto no es tomado en cuenta por la mayoría de las instituciones educativas, las que muchas veces ignoran el conocimiento y la experiencia de los profesores de su institución, pues asumen e implementan modelos, investigaciones y propuestas externas; generadas por investigadores externos a los problemas íntimos de la propia institución (Dávila, et al., 2013).

Del mismo modo, existen varios modelos de GTH (Ver tabla 2), que permiten a los interesados interactuar de manera interna y externa en la organización, así como también satisfacer sus necesidades acordes a los intereses comunes. A continuación, se demuestra mediante una tabla los diferentes modelos de GTH propuesto por (Mondragón et al., 2017; Cruz et al., 2002). 


\section{Journal of the Academy $|127|$}

\section{Ideas claves sobre la práctica profesional técnica}

El paradigma técnico en la formación docente busca vincular el desempeño del educador con el rendimiento académico del estudiante. El perfil profesional del "profesor técnico" se estructura en torno al concepto de eficacia y/o competencia orientada a formar docentes especialistas en diseñar alternativas para atender las exigencias que plantee cualquier situación educativa.

Es una propuesta restringida y reduccionista, porque solo busca establecer cuanto y cuántos contenidos se han aprendido y no qué tipos de aprendizajes se han estimulado, su propósito final es promover un aprendizaje memorístico. Por consiguiente, un profesor eficaz es aquel que resuelve problemas en el aula de manera rutinaria aplicando reglamentos, guías o manuales.

Entre los rasgos que definen la práctica docente técnica se encuentran: una formación mesológica (se pone énfasis en los medios didácticos), acentuación en el tecnicismo (priorización de métodos y técnicas didácticas), el utilitarismo (priorización del conocimiento dirigido a resolver problemas), neutralidad en el quehacer, análisis y descomposición de los métodos de enseñanza y aprendizaje. Desarrollar una enseñanza externa y alejada a las necesidades e intereses de los estudiantes, el perfil profesional se ha reducido a "ser un transmisor acrítico de información y finalmente, un docente alienado" (Vergara, 2016).

Otro rasgo "malicioso" de la práctica técnica se muestra en el distanciamiento del docente de la problemática vital del aula. Este enfoque privilegia la investigación educativa cuantitativa orientada a investigar problemas que no son vitales al aula y, cuyos resultados se presentan en una acumulación de tablas, datos e índices estadísticos. Los procesos de docencia e investigación no solamente deben estar separados; sino que se considera que ambos son incompatibles entre sí.

La fundamentación teórica que sustenta el paradigma técnico es la "pragmática- conductista" que en los esencial postula una formación docente centrada en la "eficacia y/o competencia", en la adquisición de dos competencias básicas: la programación por contenidos y la aplicación de técnicas e instrumentos de evaluación para medir el nivel de aprendizaje de los contenidos desarrollados (Araujo, 2013). 


\section{Journal of the Academy $|128|$}

En este contexto, las funciones que cumple el docente técnico se reducen esencialmente a organizar, disponer y consumir los conocimientos producidos por otros, lo que lleva a la no cualificación y pérdida de autonomía de manera progresiva. El desarrollo de su experiencia se da en torno a las recomendaciones de los expertos en currículo, enseñanza, administración y en evaluación; quienes son los que piensan; mientras que los profesores son reducidos al papel de simples ejecutores de esos pensamientos y conocimientos.

La consecuencia de todo esto es que no únicamente se les aparta de los procesos de producir conocimiento pedagógico práctico como: sistematización, deliberación y reflexión en y sobre la práctica; sino que, toda su experiencia en aula se convierte en una experiencia rutinaria. Como consecuencia de esta visión la "función docente" solamente se centra en desarrollar habilidades dirigidas al "como" enseñar.

\section{METODOLOGÍA}

La metodología de investigación desarrollada en la presente investigación fue de tipo cuantitativa-cualitativa-reflexiva, diagnóstica.

La población de estudio estuvo conformada por 90 estudiantes del programa académico de Educación Artística de la Escuela Superior de Formación Artística Pública Bellas Artes Macedonio de la Torre, distribuidos en cinco ciclos académicos. La muestra estuvo constituida por 16 estudiantes, los que fueron seleccionados de acuerdo a los siguientes criterios: Que asistieran al curso de práctica profesional docente final durante el X ciclo académico 2019-II, que se encontraran en la fase de internado desarrollando clases en los colegios de la zona, que hubieran concluido todos los cursos teóricos y talleres de ejecución artística y, finalmente, que estuvieran ejecutando su proyecto de tesis de titulación y por 2 docentes responsables de los cursos de práctica profesional.

En el recojo y análisis de datos se combinaron técnicas e instrumentos cuantitativos y cualitativos y así obtener una visión global sobre el desarrollo de la práctica docente. En la modalidad cuantitativa se aplicó la técnica de la escala de Likert y el instrumento del cuestionario y en la modalidad cualitativa se aplicó la técnica de observación participante y el instrumento de la guía de observación y análisis de portafolios de práctica. La guía de observación y análisis fue validada a través de la técnica de “Juicio de expertos”, mientras que 


\section{Journal of the Academy | 129 |}

la confiabilidad del cuestionario de la escala de Likert se determinó mediante el método AlfaCron Bach, obteniéndose un resultado de 0.86 de confiabilidad.

Los resultados cuantitativos del cuestionario de la escala se obtuvieron mediante el programa SPSS 26, mientras que los resultados cualitativos se obtuvieron a través de las técnicas de sistematización - reflexión y el desarrollo de guías de sistematización y reflexión. Los resultados finales se obtuvieron a través de la triangulación de sujetos e instrumentos con la participación de los sujetos de la muestra.

\section{RESULTADOS}

Resultados se presentan en dos dimensiones: los obtenidos de la aplicación de la escala de Likert o resultados cuantitativos y los obtenidos a través de las asambleas de sistematización reflexión o resultados cualitativos.

\section{Resultados obtenidos a través de la aplicación de la escala de Likert a los estudiantes}

\section{Tabla 1}

Concepciones de los estudiantes sobre las prácticas profesionales

\begin{tabular}{llllll}
\hline $\mathrm{N}^{\circ}$ & Criterios básicos & $\begin{array}{l}\text { En } \\
\text { desacuerdo }\end{array}$ & $\begin{array}{l}\text { total } \\
\text { desacuerdo }\end{array}$ & $\begin{array}{l}\text { En } \\
\text { acuerdo }\end{array}$ & $\begin{array}{l}\text { En } \\
\text { acuerdo }\end{array}$ \\
\hline 1 & $\begin{array}{l}\text { Dictar clases o sesiones de } \\
\text { aprendizaje en las aulas. }\end{array}$ & 17 & 8 & 42 & 33 \\
2 & $\begin{array}{l}\text { Aplicar métodos, técnicas y } \\
\text { recursos didácticos. }\end{array}$ & 8 & 8 & 42 \\
3 & $\begin{array}{l}\text { Desarrollar nuestras clases } \\
\text { utilizando esquemas y modelos } \\
\text { aprobados por la institución. }\end{array}$ & 0 & 0 & 17 & 83 \\
$4 \quad \begin{array}{l}\text { Desarrollar las clases de acuerdo } \\
\text { a la aprobación de los docentes de }\end{array}$ & 0 & 0 & 8 & 92 \\
$\begin{array}{l}\text { aula y de práctica. } \\
\text { Cumplir con la programación }\end{array}$ & 17 & 8 & 33 & 42 \\
anual y de unidad. & $\begin{array}{l}\text { Aplicar lo aprendido en los ciclos } \\
\text { previos de la carrera. }\end{array}$ & 0 & 0 & 17 & 83
\end{tabular}


$7 \quad$ Experiencias que nos ayudan a

formarnos de manera personal y

profesional como futuros

profesores.

8 Identificar y explicar los

fundamentos teóricos que

subyacen en la práctica

profesional.

9 Permite articular y vincular teoría 28

- práctica en nuestras clases.

10 Cuestionar las acciones realizadas

para mejorar la calidad de nuestras clases.

11 Asumir el error no como una

forma de castigo; sino como

oportunidad para mejorar

continuamente nuestra práctica pedagógica.

12 Permite ser autónomos y tomar

posiciones y decisiones de forma

libre durante nuestras clases.

13 Investigar los incidentes críticos

surgidos en el aula y situaciones

en la institución educativa.

14 Proponer y aplicar alternativas de

solución a los problemas

pedagógicos identificados.

15 Reflexionar conjuntamente con

los estudiantes promueve la

efectividad de las experiencias

didácticas experimentadas.

16 Discutir y compartir con los

demás (pares) las experiencias realizadas durante la práctica.

17 El trabajo cooperativo es una 


\section{Journal of the Academy $|131|$}

solucionar problemas en el aula e

institución educativa.

18 Escuchar las críticas de docentes, 50

8

formadores y compañeros para

asumir decisiones y mejorar

como docentes.

Fuente: Respuestas de los estudiantes a la escala de Likert aplicada. Elaboración propia.

En la tabla 1, se muestra que los estudiantes eligieron en un alto porcentaje las valoraciones de “Acuerdo o Totalmente de Acuerdo" en un $80 \%$ en los siguientes criterios básicos: dictar clases o sesiones de aprendizaje en las aulas, aplicar métodos, técnicas y recursos didácticos, desarrollar nuestras clases utilizando esquemas y modelos aprobados por la institución, desarrollar las clases de acuerdo a la aprobación de los docentes de aula y de práctica, cumplir con la programación anual y de unidad y aplicar lo aprendido en los ciclos previos en la carrera. Mientras que a los demás criterios básicos le asignan una valoración por encima del $50 \%$ entre "Total en desacuerdo y en desacuerdo".

\section{Tabla 2}

Rutinas o actividades desarrolladas en las prácticas profesionales

\begin{tabular}{|c|c|c|c|c|c|c|}
\hline $\mathbf{N}^{\circ}$ & Principales actividades y rutinas & Nunca & $\begin{array}{l}\text { A } \\
\text { veces }\end{array}$ & $\begin{array}{l}\text { Raras } \\
\text { veces }\end{array}$ & $\begin{array}{l}\text { Casi } \\
\text { siempre }\end{array}$ & Siempre \\
\hline 1 & $\begin{array}{l}\text { Solicitar los contenidos y capacidades a los } \\
\text { docentes de aula para programar nuestras } \\
\text { clases. }\end{array}$ & 0 & 0 & 0 & 0 & 100 \\
\hline 2 & $\begin{array}{l}\text { Presentar nuestros diseños de clase para su } \\
\text { revisión y aprobación. }\end{array}$ & 0 & 0 & 0 & 0 & 100 \\
\hline 3 & $\begin{array}{l}\text { Recibir visitas de monitoreo y } \\
\text { acompañamiento en el aula por el docente } \\
\text { de práctica. }\end{array}$ & 20 & 50 & 30 & 0 & 0 \\
\hline 4 & $\begin{array}{l}\text { Revisión de los diseños y planes de clase } \\
\text { por el docente de aula y de práctica. }\end{array}$ & 0 & 0 & 0 & 0 & 100 \\
\hline 5 & $\begin{array}{l}\text { Observar y reflexionar sobre sesiones de } \\
\text { aprendizaje demostrativas desarrolladas por } \\
\text { el docente de práctica. }\end{array}$ & 100 & 0 & 0 & 0 & 0 \\
\hline
\end{tabular}


6 Ayudar al maestro/a de aula en la atención

$0 \quad 0 \quad 0$

77

de los estudiantes.

7 Presentar los portafolios (archivadores) cada 0

$0 \quad 0$

100

fin de ciclo para su revisión y calificación.

8 Programar y ejecutar el proceso de

$\begin{array}{llll}0 & 0 & 0 & 77\end{array}$

evaluación a los estudiantes.

Registrar información relevante en nuestros

9 diarios de práctica, sobre estrategias de

68

32

0

0

0

enseñanza y aprendizaje desarrolladas en

aula.

Desarrollar reuniones permanentes de

10 sistematización de experiencias realizadas

70

30

0

0

0

para mejorarlas.

Desarrollar actividades de reflexión para

11

solucionar problemas pedagógicos

sistematizados y así mejorar nuestra práctica

80

20

0

0

0

en el aula.

Recibir realimentación y acompañamiento

del docente de aula y del docente formador

de práctica para mejorar el proceso de

0

50

0

evaluación.

Desarrollar procesos de autoevaluación y

13

evaluación de pares de experiencias

significativas desarrolladas en nuestras

100

0

0

0

0

prácticas.

Desarrollar actividades permanentes de

14 organización de nuestro portafolio de

100

0

0

0

0

práctica.

15

Participar del taller de sistematización cada fin de ciclo.

100

0

0

0

0

Fuente: Respuestas de los estudiantes a la escala de Likert aplicada. Elaboración propia. 
La tabla 2, muestra que los estudiantes asignaron un alto porcentaje a las valoraciones de "Nunca, A Veces y Raras veces" a las siguientes actividades y rutinas: recibir visitas de monitoreo y acompañamiento en el aula por el docente formador de práctica, observar y reflexionar sobre sesiones de aprendizaje demostrativas desarrolladas por el docente formador, registrar información relevante en los diarios de práctica sobre estrategias de enseñanza y aprendizaje desarrollas, desarrollar reuniones permanentes de sistematización de experiencias realizadas para mejorarlas, desarrollar actividades de reflexión para solucionar problemas pedagógicos sistematizados, desarrollar procesos de autoevaluación y evaluación de pares y de experiencias significativas desplegadas en las prácticas, desarrollar actividades permanentes de organización del portafolio de práctica y participar del taller de sistematización cada fin de ciclo.

Mientras que las actividades y rutinas de: solicitar los contenidos y capacidades a los docentes de aula para programar clases, presentar diseños de clase para su revisión y aprobación, revisión de diseños y planes de clase por el docente de aula y de práctica, presentar los portafolios de práctica cada fin de ciclo para su revisión y calificación, ayudar al maestro/a de aula en la atención de los estudiantes y programar y ejecutar el proceso de evaluación a los estudiantes; le asignaron un alto porcentaje en las valoraciones de "Casi siempre y Siempre".

\section{Tabla 3}

Herramientas metodológicas utilizadas en el desarrollo de las prácticas profesionales

\begin{tabular}{|c|c|c|c|c|c|c|}
\hline $\mathrm{N}^{\circ}$ & $\begin{array}{l}\text { Principales herramientas } \\
\text { metodológicas }\end{array}$ & Nunca & $\begin{array}{c}\text { A } \\
\text { veces }\end{array}$ & $\begin{array}{l}\text { Raras } \\
\text { veces }\end{array}$ & $\begin{array}{c}\text { Casi } \\
\text { siempre }\end{array}$ & Siempre \\
\hline 1 & $\begin{array}{l}\text { El diario de campo como herramienta de } \\
\text { registro y reflexión. }\end{array}$ & 30 & 20 & 33 & 7 & 10 \\
\hline 2 & $\begin{array}{l}\text { Guías de observación para dinamizar el } \\
\text { proceso de registro en el diario. }\end{array}$ & 26 & 36 & 34 & 4 & 0 \\
\hline 3 & $\begin{array}{l}\text { Rúbricas y fichas de auto y evaluación de } \\
\text { pares. }\end{array}$ & 100 & 0 & 0 & 0 & 0 \\
\hline 4 & $\begin{array}{l}\text { Rúbricas y fichas de evaluación para el } \\
\text { docente de aula y docente formador. }\end{array}$ & 100 & 0 & 0 & 0 & 0 \\
\hline 5 & $\begin{array}{l}\text { Guía de sistematización de experiencias } \\
\text { educativas. }\end{array}$ & 43 & 23 & 34 & 0 & 0 \\
\hline
\end{tabular}




\section{Journal of the Academy | $134 \mid$}

Portafolio de práctica como instrumento

6 de evaluación y progreso de la formación 40

40

docente.

Guías de reflexión de y sobre la práctica

7 profesional pedagógica.

33

Instrumentos para evaluar evidencias o

8 productos de aprendizaje.

9 Matriz de evaluación con criterios e indicadores para evaluar diarios,

portafolios y sesión de aprendizaje.

Protocolos para observar, registrar y

10 reflexionar la práctica pedagógica.

Guías de análisis de contenidos tanto

11 cualitativos como cuantitativos.

60

Programa Excel para organizar y

procesar datos recogidos durante la

100

40

27

0

0

$\begin{array}{lllll}0 & 40 & 26 & 34 & 0\end{array}$

$\begin{array}{lllll}73 & 27 & 0 & 0 & 0\end{array}$

$\begin{array}{lllll}74 & 26 & 0 & 0 & 0\end{array}$

12 práctica.

Programa SPSS para procesar datos

13 recogidos en el desarrollo de la práctica.

$\begin{array}{lllll}100 & 0 & 0 & 0 & 0\end{array}$

Internet para buscar y seleccionar

información de forma adecuada y

40

$14 \quad 20 \quad 27$

14 oportuna.

Fuente: Respuestas de los estudiantes a la escala de Likert aplicada. Elaboración propia.

En tanto que en la tabla 3, se manifiesta un porcentaje muy alto en las valoraciones "Nunca, A Veces y Raras veces" en cuanto a manejo de herramientas metodológicas utilizadas por los estudiantes en el desarrollo de las prácticas profesionales.

\section{Resultados obtenidos a través de la triangulación de sujetos e instrumentos}

Los resultados en la modalidad cualitativa, se obtuvieron en dos momentos: en el primero se realizó el análisis cualitativo de categorización y teorización y en el segundo momento estos resultados se triangularon con las estudiantes y docentes de la muestra en una asamblea reflexiva. Los resultados finales se organizaron bajo la forma de reflexiones $(\mathrm{R})$ y se presentan a continuación. 


\section{Journal of the Academy $|135|$}

\section{Concepciones sobre la práctica profesional}

Las concepciones y significados de los estudiantes practicantes reflejan sus creencias, lo que ellos entienden por práctica profesional, sus valoraciones sobre lo que hacen y lo que vivencian; todo esto expresado a través de sus opiniones y reflexiones.

Dictado de clases. "Dictar nuestras clases en los colegios donde realizamos nuestras prácticas" (R1). "Desarrollar clases con los niños en las aulas" (R2). "Aplicar en las clases los contenidos que nos asignan los profesores de aula (R3). "Desarrollar clases bajo el asesoramiento del profesor de práctica de la Escuela" (R4) y "Dictar los contenidos para que los estudiantes los aprendan, en la clase" (R5).

Desarrollar las capacidades. "Los profesores de aula nos designan las capacidades a desarrollar en cada clase" (R1). "El docente de aula a veces nos da la programación de unidad para que nosotras seleccionemos las capacidades a desarrollar" (R2). "Hay semanas que no he dictado clase porque los profesores no me dieron las capacidades" (R3).

Aplicación de lo aprendido en los ciclos anteriores. "En las clases aplicamos lo que hemos aprendido en los ciclos anteriores acá en la Escuela" (R1). "Los contenidos trabajados en los talleres de didáctica nos sirven mucho para nuestra práctica" (R2). "Las técnicas artísticas trabajadas en los talleres nos sirvieron mucho para atender a los niños y niñas en nuestras clases" R3).

Desajuste entre el aprendizaje recibido en la Escuela y la realización de las prácticas. "Lo que nos enseñan en los talleres integrados no se aplica mucho en nuestras prácticas" (R1). "Las técnicas artísticas que nos enseñaron en los talleres no nos sirvieron de mucho para enseñar a los niños y niñas" (R2). "No nos enseñan técnicas de teatro, música y danzas folclóricas en los talleres" (R3).

Aplicación de lo que los profesores de aula y docente de práctica quieren. "Tenemos que hacer lo que la profesora nos ha revisado" (R1). "Si el diseño no ha sido revisado por el profesor de práctica no podemos dictar la clase" (R2). "A veces el profesor nos cambia todo el diseño" (R3). "La profesora de aula es la única que nos evalúa la ejecución de la clase" (R4).

Desarrollar contenidos. "Tenemos que desarrollar los contenidos que nos dan los profesores de aula" (R1). "Tenemos que dictar los contenidos que nos revisan los profesores de práctica" (R2). "A veces, a los profesores de aula más les interesan los contenidos que desarrollar capacidades" (R3). 


\section{Journal of the Academy $|136|$}

Proceso para desarrollar y mejorar la práctica profesional. Las tareas o actividades que ayudan a reflexionar sobre nuestras prácticas, son las siguiente: "La presentación de tareas de los estudiantes" (R1)." Evaluar los aprendizajes de los estudiantes" (R2). "Compartir los aprendizajes de los niños" (R3). "Los cuadros que ejecutan los niños en las clases" (R4). "La planificación de las sesiones de aprendizaje" (R5) y "Las sugerencias y correcciones de los profesores" (R6).

\section{Rutinas o actividades que se desarrollan durante las prácticas profesionales}

Las rutinas y/o actividades identificadas y propuestas por las estudiantes practicantes fueron las siguientes:

Programación de la sesión. "Pedir a los profesores de aula los contenidos, actividades o capacidades a desarrollar en la clase" (R1). "Programar la clase siguiendo el "esquema" que nos dan los profesores de práctica, el cual lo utilizamos como plantilla" (R2). "Presentación de nuestro diseño a la profesora de práctica para su revisión y corrección" (R3). "Si no se presenta el diseño para que la profesora de práctica lo revise no podemos desarrollar esa clase" (R4).

Organización del portafolio. "Archivar todos los diseños de clase desarrollados y firmados por la profesora de práctica" (R1). "Archivar las evaluaciones y materiales utilizados en cada clase" (R2). "Solicitamos el PEI y programaciones de grado al docente de aula y luego los archivamos en el portafolio" (R3). "Fotocopiamos el reglamento de práctica y lo archivamos" (R4).

Llenar el cuaderno de práctica. "Adquirimos un cuaderno A4" (R1). "Escribimos ocurrencias y anécdotas sucedidos en clase" (R2). "El llenado del cuaderno lo hacemos de acuerdo a nuestro parecer, ya que no tenemos orientaciones por parte del docente" (R3). "Presentamos nuestro cuaderno a la docente de práctica para su revisión y nos evalúe" (R4).

Ayudar al profesor (a) en el aula de clases. "Participar en las actuaciones que se realizan en la institución o colegio" (R1). "Ayudar al profesor (a) de aula en las actividades artísticas programadas” (R2). “Ayudar a controlar a los niños mientras el profesor de aula desarrolla su clase" (R3).

\section{Herramientas e instrumentos utilizados durante las prácticas profesionales}

Las herramientas o instrumentos identificados por los estudiantes practicantes fueron los siguientes:

Esquema de programación de la sesión. "Es un esquema de sesión de aprendizaje que nos entrega la profesora de práctica, el cual tenemos que seguir en cada una de nuestras sesiones" 
(R1). "Es un modelo que nos da la profesora para utilizarlo en las programaciones de nuestras sesiones de aprendizaje" (R2). "En las clases de didáctica hemos utilizados dichos modelos" (R3).

Modelos y esquemas bajados de internet. "Para las prácticas o ejercicios de los estudiantes siempre utilizamos modelos y figuras que bajamos de internet" (R1). "Las siluetas de figuras, animales y plantas los descargamos de internet, lo imprimimos y entregamos a los estudiantes" (R2). "Cuando dejamos trabajo a los estudiantes ellos siempre nos presentan esquemas bajados de internet" (R3).

El portafolio de práctica. "Es un archivador grande donde archivábamos los documentos de la práctica como sesiones de aprendizaje, evaluaciones aplicadas y materiales utilizados" (R1). "Se entrega al finalizar el ciclo a la profesora para su calificación" (R2). "Desconocemos la forma y los criterios de cómo nos califican nuestro portafolio" (R3).

Modelos de evaluación. "Para evaluar los trabajos de los estudiantes utilizamos formatos de instrumentos que los cuales lo aplicamos como plantilla" (R1). "Los instrumentos de evaluación que utilizamos generalmente los conseguimos de internet” (R2).

\section{Concepciones y valoración sobre las experiencias de sistematización, crítica y reflexión desarrolladas durante las prácticas profesionales}

Las concepciones y valoraciones que dieron las estudiantes fueron las siguientes:

La crítica dentro de la práctica profesional. "Es emitir nuestras opiniones sobre una cuestión específica" (R1). "La crítica se debe realizar en todas las clases y cursos del plan de estudios, pero no es así" (R2). "A través de la crítica observamos, analizamos y encontramos sus causas y efectos de situaciones o casos específicos" (R3). "La aplicación de crítica ayuda a mejorar nuestras prácticas" $(\mathrm{R} 4)$.

La reflexión. "Es obtener conclusiones sobre un tema tratado o desarrollado en clase" (R1). "Consiste en buscar e identificar lo bueno y malo de una situación estudiada" (R2). "Es la capacidad de pensar un tema o situación específica y establecer si es bueno o malo" (R3).

Experimentación en la práctica profesional. "Creo que nunca hemos aplicado estas técnicas en el desarrollo de los cursos de práctica" (R1), "Realmente los profesores no nos hacen razonar, criticar y reflexionar sobre lo que hacemos en nuestras prácticas" (R2). "Ni siquiera en los talleres principales nos hacen que evaluemos nuestro propio trabajo para luego mejorarlo" (R3). 


\section{DISCUSIÓN DE RESULTADOS}

Sobre las concepciones de las prácticas profesionales pedagógicas, en las expresiones, opiniones y valoraciones dadas por los estudiantes, se puntualiza que la práctica profesional pedagógica significa: dictar clases, aplicar lo aprendido en los ciclos anteriores en el plan de estudios, aplicar lo que los profesores de aula y docente de práctica quieren o exigen y desarrollar contenidos pedagógicos previamente asignados por los profesores de aula. Así mismo, se afirma que existe un desajuste entre los aprendizajes recibidos en los cursos y talleres artísticos en la Escuela y la realización de las prácticas pedagógicas y que el desarrollo de las prácticas profesionales pedagógicas no ayudan a mejorar y cualificar las mismas.

Resulta complejo y metódico, identificar a qué enfoque pertenecen las concepciones sobre la práctica develadas; ya que en el ejercicio profesional conviven ambas perspectivas: la técnica y la reflexiva. Por un lado, se puntualiza que la práctica profesional requiere de un profesor experto en estrategias, métodos y técnicas didácticas; por otro, de un profesor crítico, autónomo, flexible y atento que advierta las necesidades y exigencias del aula, la escuela y de la propia comunidad. Sin embargo, las concepciones develadas guardan relación con el estudio realizado por Ávila y Pasek, (2013) que reportó que las concepciones de los docentes sobre la educación para el trabajo y la práctica profesional pedagógica se aproximaban a una concepción tradicional-instrumentalista y conductista caracterizada por estar limitada exclusivamente al dictado de contenidos exigidos por un programa o asignatura, sin tomar en cuenta los intereses de los estudiantes, ni la construcción de proyectos de vida, la promoción de actividades concernientes al trabajo en el hogar, la escuela y la comunidad.

Los estudiantes en su mayoría identifican las siguientes rutinas o actividades inherentes al ejercicio de la práctica profesional pedagógica: la programación de las sesiones de aprendizaje se realiza a través de esquemas o formatos a manera de plantillas, la organización del portafolio y el llenado del cuaderno de práctica siempre se realiza al concluir el ciclo académico y sin la ayuda del profesor (a). Las rutinas o actividades identificadas en el desarrollo de la práctica profesional pedagógica, se adscriben a una práctica tradicional caracterizada por el protagonismo del docente y la trasmisión de contenidos.

Desde las propias experiencias de los estudiantes, la crítica y la reflexión son procesos medianamente conocidos por ellos, pero escasamente experimentados; capacidades como: 


\section{Journal of the Academy $|139|$}

establecer criterios de acuerdo con el contexto, ponerse en evidencia, autocorregirse y la formación de juicios para consolidar el pensamiento crítico y reflexivo, es un camino desconocido. Estas valoraciones y experiencias de los estudiantes guardan relación con el estudio realizado por el Ministerio de Educación, (2016) que consistió en el monitoreo en aula a 324 docentes formadores correspondientes a 76 Institutos de Educación Superior Pedagogos públicos y a 125 estudiantes de Formación Inicial Docente a nivel nacional. El resultado de dicho estudio confirmó que escasamente el $20.3 \%$ de docentes monitoreados desarrollaba actividades en el aula que promovían el pensamiento crítico en los estudiantes.

Finalmente, de las experiencias de reflexión, crítica y sistematización desarrolladas en las prácticas profesionales se comprueba la existencia de mínimas y fortuitas experiencias de aprendizaje reflexivo y crítico en el desarrollo de las mismas; no hay un mínimo esfuerzo por estimular el aprendizaje reflexivo, orientado a facilitar el desarrollo de habilidades de deliberación, argumentación, la toma de decisiones y proponer soluciones creativas a la problemática surgida en las aulas e instituciones educativas.

Según las concepciones y valoraciones dadas por los estudiantes, la práctica profesional que se desarrolla, no es un espacio para crear conocimientos prácticos a partir del análisis crítico y reflexión sobre lo que acontece en las aulas, como resultado tanto de un ejercicio individual, como cooperativo, por parte de los estudiantes y docentes. No se experimentan estrategias de aprendizaje reflexivo centradas en el diálogo, la indagación, resolución de problemas y la innovación.

Los resultados obtenidos en el estudio, guardan relación con los hallazgos del estudio de Mata, et al. (2018), concretamente con los siguientes:

- Los docentes se preocupan más por enseñar contenidos, que promover un entorno favorable para promover el pensamiento crítico, la aproximación a la comprensión y construcción de aprendizajes para luego transformar.

- Los conocimientos que se promueven se caracterizan por una desarticulación total de la teoría con su aplicación práctica, lo cual desmotiva al estudiante en su proceso de aprendizaje. 


\section{Journal of the Academy $|140|$}

- La indagación, investigación y reflexión como herramientas metodológicas de aprendizaje son escasamente utilizadas en las aulas, ignorando por completo que son herramientas esenciales para promover el aprendizaje crítico y reflexivo.

La consecuencia de esta situación es que no se desarrolla una epistemología de la práctica reflexiva cuya esencia es alentar la reflexión como elemento transformador de la propia práctica del profesor y así formar un docente con capacidad de improvisar durante su ejercicio profesional, improvisación que no proviene del azar, sino de su experiencia vital, de las interacciones con sus estudiantes, con sus pares y de sus propias estructuras mentales, epistemológicas e intuitivas desarrolladas a través de su propia experiencia, pero que tiene que ser articuladas de forma unitaria al momento de ejercer la profesión docente (Ruffinelli; 2017).

Finalmente, la implicancia de la perspectiva técnica en la formación actual de los estudiantes de Educación Artística de artes plásticas, condiciona a estos para que al momento de desarrollar sus prácticas en las aulas aprendan a ser maestros asumiendo los estilos de enseñanza e imitando roles y acciones de los maestros que tienen como modelos, así como asimilar la cultura escolar con todas sus implicaciones y cumplir con las normas y criterios establecidos de antemano; en lugar de reconstruir sus propias prácticas y percepciones, así como de las estructuras organizacionales institucionales y entender su funcionamiento para proponer transformaciones creativas de las mismas.

\section{CONCLUSIONES}

Concluida la investigación, podemos afirmar que se lograron los objetivos propuestos. El análisis de los datos obtenidos nos ha permitido arribar a las siguientes conclusiones:

Las concepciones develadas, las rutinas, actividades y herramientas metodológicas identificadas por los estudiantes, determinan que la práctica profesional pedagógica que actualmente se desarrolla se adscribe dentro del enfoque técnico - academicista.

La crítica y reflexión como competencias fundamentales que fomentan la argumentación, crítica, reflexión, la toma de decisiones y solución de problemas son escasamente promovidas y alentadas durante las enseñanza - aprendizaje de los cursos de práctica pedagógica pre profesionales; por el contrario, se priorizan otras formas de enseñanza y el uso de herramientas 


\section{Journal of the Academy $|141|$}

metodológicas dejando de lado otras formas de enseñanza - aprendizaje que favorecen la formación y desarrollo de competencias profesionales pedagógicas: prácticas reflexivas.

Las experiencias pedagógicas - didácticas de manejo de herramientas metodológicas que favorezcan la crítica y reflexión en la práctica profesional son escasas; lo que genera desempeños rutinarios e imitativos durante el desarrollo de las mismas, ignorando estos espacios como escenarios de acción, transformación, innovación y mejora.

Finalmente, los resultados obtenidos abren la posibilidad de seguir profundizando el estudio, mediante la ampliación de la muestra de estudiantes e incorporando a docentes responsables de práctica en las Escuelas de Arte y de las instituciones educativas donde los estudiantes realizan sus prácticas y así obtener resultados más completos y confiables que permitan proponer y ensayar alternativas integrales de solución y mejora de la práctica profesional pedagógica.

\section{REFERENCIAS BIBLIOGRÁFICAS}

Anijovich, R. y Capelletti, G. (2018). La práctica reflexiva en los docentes en servicio. Posibilidades y limitaciones. Revista de Educación, (28), 7492. https://www.redalyc.org/articulo.oa?id=384555587005

Araujo, R. (2013). Teorías contemporáneas del aprendizaje. EDIMAG.

Ávila de Vanegas, N. y Pasek de Pinto, E. (2013). Concepciones de la educación para el trabajo desde los 271-285. https://www.redalyc.org/articulo.oa?id=35630152012

Carbajal, N. (2013). Formación de profesores prácticos reflexivos mediante la articulación de la investigación acción y la práctica profesional reflexiva. [Tesis Doctorado, Universidad Nacional de Trujillo]. https://dspace.unitru.edu.pe

Cárdenas, N. (2015) Análisis crítico del sistema de evaluación del desempeño profesional del docente chileno: Un estudio en la comunidad educativa de la ciudad de Osorno Chile. [Tesis doctorado, Universidad Autónoma de Barcelona]. https://www.tdx.cat/bitstream/handle/10803/384556/necs1de1

Dávila, G., Balcarce, F., Comelin, A., Calderón, M. y Varela, P. (2013). Conocimiento práctico de los profesores: sus características y contradicciones en el contexto universitario actual. Revista de la educación superior. 42(166). 37-56. http://www.scielo.org.mx/scielo.php?script=sci_arttext\&pid=S0185$\underline{27602013000200002}$ 
Domingo, A. (2015) Práctica reflexiva para docentes. De la reflexión ocasional a la reflexión metodológica. Editorial Publicia. https://practicareflexiva.pro/wpcontent/uploads/2015/09/Practica-reflexiva-para-docentes.pdf

Domingo, A. (2017). La práctica reflexiva, un nuevo modelo de formación docente. Entrevista publicada en Eduprensa expertas. https://practicareflexiva.pro/wpcontent/uploads/2019/03/

Lever, C. y López, K. (2019). La práctica docente reflexiva en profesores mexicanos ante los retos de la nueva ciudadanía. Education Siglo XXI, (37), 113-130. https://doi.org/10.6018/educatio.363421.

Lupion, T. y Gallego, M. (2017). Compartiendo la mirada: una experiencia en práctica reflexiva para formación permanente. Revista Electrónica Interuniversitaria de Formación del Profesorado, 20(1), 127-144. http://dx.doi.org/10.6018/reifop.20.1.244931

Mata, A., Hernández, P. y Centeno. G. (2018). La práctica reflexiva en los docentes de posgrado, comprender para transformar. INNOEDUCA. International Journal of Tech Nology and Education al Innovation, 4(1), 36-43 http://dx.doi.org/10.24310/innoeduca.2018.v4i1.3594.

Ministerio de Educación (2016). Diseño Curricular Básico Nacional de la Formación Inicial Docente. Programa de Estudios de Educación Inicial. Ministerio de Educación.

Rojas, A. (2015). Retos a la Educación Peruana en el Siglo XXI. REICE. Revista Iberoamericana sobre Calidad, Eficacia y Cambio en Educación, 14(1), 101-115. https://www.redalyc.org/jatsRepo/551/55143412006/html/index.html

Ruffinelli, A. (2017). Formación de docentes reflexivos: un enfoque en construcción y disputa. Pesquisa. 43(1), 97-111. http://dx.doi.org/10.1590/S1517-9702201701158626

Salinas, Á., Chandía, E., y Rojas, D. (2017). Validación de un instrumento cuantitativo para medir la práctica reflexiva de docentes en formación. Estudios pedagógicos, 43(01), 289309. http://dx.doi.org/10.4067/S0718-07052017000100017

Souto, M. (2016). Pliegues de la formación. Sentido y herramientas para la formación docente. Homo Sapiens. Ediciones Rosario.

Vergara, M. (2016). La práctica docente. Un estudio desde los significados. Revista CUMBRES, 2(1), 73 - 99. https://dialnet.unirioja.es/servlet/articulo?codigo=6550779 